\title{
Padronização do gasto metabólico de repouso e proposta de nova equação para uma população feminina brasileira
}

\author{
Characterization of metabolic resting rate and proposal \\ of a new equation for a female Brazilian population
}

Alessandra E. Rodrigues', Marcio C. Mancini', Lorença Dalcanale', Maria Edna de Melo', Cíntia Cercato', Alfredo Halpern'

${ }^{1}$ Grupo de Obesidade e Síndrome Metabólica, Disciplina de Endocrinologia e Metabologia, Hospital das Clínicas da Faculdade de Medicina da Universidade de São Paulo (HC-FMUSP), São Paulo, SP, Brasil
Correspondência para: Alessandra E. Rodrigues Rua Aleixo Garcia, 40, ap. 41 04545-010 - São Paulo, SP, Brasil sna.nutri@uol.com.br

Recebido em 13/Set/2009 Aceito em 5/Abr/2010

\section{RESUMO}

Objetivos: Os objetivos do estudo foram normatizar o gasto metabólico de repouso (GMR) de mulheres brasileiras, avaliar a adequação da fórmula de Harris Benedict (HB), a relação entre suas variáveis e propor novas equações para estimativa do GMR. Sujeitos e métodos: Para tanto, foram utilizados 760 exames de calorimetria indireta $(\mathrm{Cl})$ de mulheres com idade entre 18 e 65 anos. Os resultados foram tabulados, a população foi dividida de acordo com quintis de peso e, então, realizaram-se as análises estatísticas. Resultados: A média de GMR variou entre 1.226 calorias/dia a 1.775 calorias/dia. A fórmula de HB superestimou o GMR em torno de $7 \%$. Concluímos, a partir da correlação entre as variáveis, que o peso possui correlação positiva com o GMR, e a idade, uma correlação negativa. Conclusão: GMR assim como coeficiente respiratório (QR) podem ser utilizados como preditores de obesidade. Nosso estudo trouxe duas novas propostas de equações, uma para a população com índice de massa corpórea (IMC) > 35 $\mathrm{kg} / \mathrm{m}^{2}$ e outra para a população com IMC $\leq 35 \mathrm{~kg} / \mathrm{m}^{2}$. Arq Bras Endocrinol Metab. 2010;54(5):470-6

\section{Descritores}

Obesidade; calorimetria indireta; gasto metabólico de repouso; fórmula de Harris Benedict

\section{ABSTRACT}

Objetives: The goals of this study were to standardize resting metabolic rate (RMR) in the Brazilian female population and evaluate the suitability of the HB equation. Subjects and methods: In order to do so, 760 indirect calorimetry $(\mathrm{Cl})$ measurements performed in Brazilian female patients between 18 and 65 years old, were used. The results were tabulated, the population distributed according to the quintiles of weight, and the statistical analyses applied. Results: The average RMR varied from 1,226 to 1,775 calories/day. The HB equation overestimated the RMR by about $7 \%$. From the correlation between the variables, we conclude that weight has a positive correlation with RMR and age a negative correlation. Conclusion: The RMR and respiratory quotient $(\mathrm{QR})$ can be used as obesity predictors. Two new equations were proposed in our study, one for the population with body mass index $(\mathrm{BMI})>35 \mathrm{~kg} / \mathrm{m}^{2}$ and another for the population with $\mathrm{BMI} \leq 35 \mathrm{~kg} / \mathrm{m}^{2}$. Arq Bras Endocrinol Metab. 2010;54(5):470-6

\section{Keywords}

Obesity; indirect calorimetry; resting metabolic rate; Harris Benedict equation

\section{INTRODUÇÃO}

I ndependentemente do fator básico desencadeador da Lobesidade, um desequilíbrio no balanço energético (BE), ou seja, entre a ingestão calórica total (IC) e o gasto energético total (GET), está intimamente relacionado à sua alta prevalência $(1)$.

O principal componente do GET é o gasto metabólico de repouso (GMR), o qual pode ser um preditor de ganho de peso (2-5). 
A forma mais comum para o cálculo do GMR na prática clínica é pelo uso de fórmulas, sendo a fórmula de Harris Benedict (HB) a mais utilizada $(6,7)$.

Entretanto, há estudos que sugerem que as fórmulas podem levar a super ou subestimação do GMR, isso porque a população estudada nem sempre corresponde à população utilizada para elaboração das fórmulas (8-12). No Brasil, HB é a equação mais utilizada, entretanto estudos indicam que existe uma superestimação em adultos de 18 a 59 anos, especialmente em mulheres $(13,14)$.

Por esse motivo, diversos autores questionam o uso de fórmulas para o cálculo do GMR e recomendam o uso da calorimetria indireta $(\mathrm{CI})$, considerada padrãoouro, ou no caso da impossibilidade da sua realização, em decorrência do seu alto custo, a criação de fórmulas específicas para a população estudada que torne os resultados mais adequados $(15,16)$.

Portanto, considerando-se esses dados, nosso estudo tem o objetivo, com base nos resultados de GMR de uma população feminina obtidos por CI, verificar se os resultados obtidos pela fórmula de $\mathrm{HB}$ realmente são superestimados, avaliar a relação das principais variáveis da CI com GMR e desenvolver uma nova fórmula para que as estimativas do GMR na população brasileira se tornem mais precisas, além de padronizar os valores de GMR para que estes possam ser utilizados como preditores de obesidade.

\section{SUJEITOS E MÉTODOS}

Inicialmente foi elaborado o Termo de Consentimento Livre e Esclarecido (TCLE). Após submissão do projeto ao Comitê de Ética, foram estudados os resultados de 1.359 exames de CI de pacientes do sexo feminino com idade de 18 a 65 anos, realizados no Serviço de Endocrinologia e Metabologia do Hospital das Clínicas da Faculdade de Medicina da Universidade de São Paulo (HC-FMUSP), de janeiro de 1995 a janeiro de 2007.

Os exames de CI de pacientes com doenças que interfiram diretamente na determinação do GMR, tais como doença inflamatória intestinal, diabetes melito, hiper ou hipotiroidismo, insuficiência cardíaca, hepática ou renal, foram excluídos, restando 760 exames.

Para a realização dos exames de CI, foi utilizado o calorímetro Deltatrac Monitor II MBM-200 (DatexEngstron Division, Instruments Corp., Helsinki, Finland) e, como recomendado, os exames foram realizados em ambiente termoneutro, com pressão, umidade e temperatura controladas, com o paciente deitado, porém vigil.

Os resultados foram então tabulados e realizou-se a análise estatística.

Para o desenvolvimento das novas equações para cálculo de GMR, a população estudada foi dividida em dois grupos: Grupo 1 (Gl), que incluiu pacientes com índice de massa corpórea (IMC) acima de $35 \mathrm{~kg} / \mathrm{m}^{2}$, e Grupo 2 (G2), que incluiu pacientes com IMC abaixo de $35 \mathrm{~kg} / \mathrm{m}^{2}$. Essa divisão foi adotada uma vez que o Grupo 1 , composto por população superobesa, apresentou resultados muito discrepantes do restante da população quando as análises estatísticas prévias foram feitas. O IMC foi escolhido por ser uma medida simples que avalia o nível de adiposidade em grandes estudos populacionais (17).

Para propor um novo modelo de cálculo do GMR, inicialmente foram estabelecidas as relações entre as variáveis: idade, peso, altura e GRM da CI, considerando o modelo de Regressão Linear Múltipla. Para avaliação da existência de relação linear, foi considerada a análise de variância e, para avaliação da importância de cada variável explicativa sobre GRM, considerou-se a medida de correlação parcial. A influência de cada caso no modelo foi avaliada considerando-se a medida de diagnóstico DFFITS (diagnóstico de regressão: identificação de dados influentes e fontes de colinearidade). Para comparação do novo modelo proposto neste estudo com a fórmula de $\mathrm{HB}$, considerou-se a estimação da equação de regressão com base em um conjunto de treinamento e efetuou-se a aplicação da equação em um conjunto de casos não considerados na estimação da equação. Neste conjunto de teste, foi também aplicada a fórmula de HB, estimando-se, assim, o resultado de GRM sob os dois modelos. Com base nos resultados, calculou-se o erro médio quadrático para a expressão calculada utilizando-se de dados da realidade brasileira e o erro médio quadrático foi obtido com a aplicação da fórmula de HB. Esse procedimento foi repetido 10 vezes, considerando-se como base para estimação do modelo $75 \%$ dos casos (conjunto de treinamento) e $25 \%$ dos casos como conjunto para teste. Valores de $\mathrm{p}<0,05$ indicaram significância estatística.

Além do novo modelo formulado, foi proposto um fator de correção para melhor ajuste da fórmula de $\mathrm{HB}$ à nossa população, por meio de um modelo de regressão considerando-se o resultado da fórmula de $\mathrm{HB}$ como variável explicativa e o valor de GMR obtido pela CI como variável resposta. A mesma validação por erro médio quadrático foi aqui aplicada. 


\section{RESULTADOS}

Inicialmente foi avaliada a influência de cada variável sobre o GMR (Tabela 1). A idade e o coeficiente respiratório $(\mathrm{QR})$ apresentaram correlações inversas com o GMR. As variáveis que apresentaram maior influência foram peso e IMC, sendo a variável peso a que se mostrou ainda mais influente.

Tabela 1. Análise da influência de cada variável sobre os valores de GMR

\begin{tabular}{lcc}
\hline Variável & $\begin{array}{c}\text { Coeficiente de } \\
\text { correlação entre a } \\
\text { variável e GMR }\end{array}$ & Valor de $\mathbf{p}$ \\
\hline Idade & $-0,224$ & $<0,001$ \\
Peso & 0,772 & $<0,001$ \\
Altura (m) & 0,268 & $<0,001$ \\
IMC & 0,698 & $<0,001$ \\
RQ (mean) & $-0,161$ & $<0,001$ \\
\hline
\end{tabular}

GMR: gasto metabólico de repouso; m: metros; mean: médio. ${ }^{*} p<0,05$

Por esse motivo a padronização do GMR foi feita dividindo-se a população em quintis de peso (Figura l, Tabela 2). Para o primeiro quintil, a média de GMR foi de 1.226 calorias/dia, para o segundo quintil, de 1.335 calorias/dia, para o terceiro quintil, de 1.409 calorias/ dia, para o quarto quintil, de 1.513 calorias/dia e, para o quinto quintil, foi de 1.775 calorias/dia.

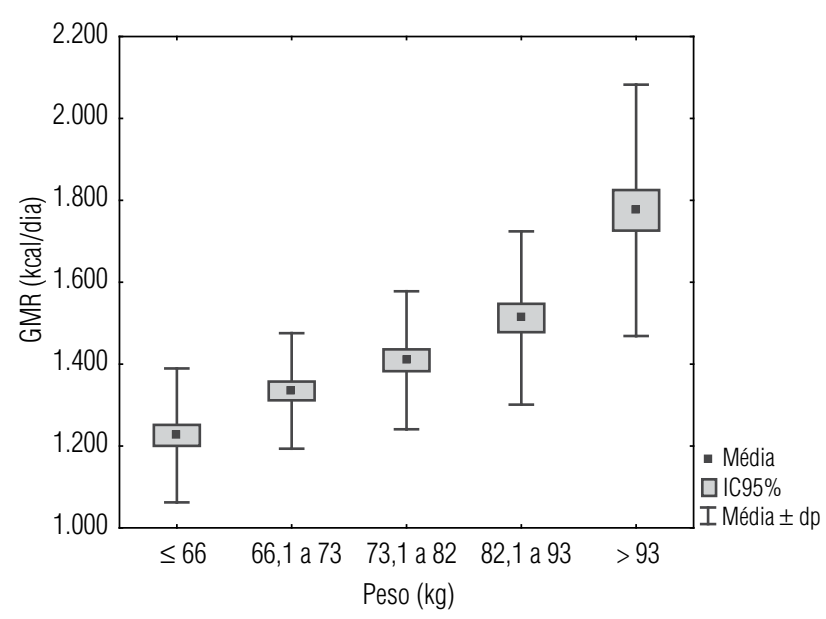

dp: desvio-padrão.

Figura 1. Padronização do GMR por quintis de peso.

A avaliação da existência de superestimação dos valores de GMR obtidos pela fórmula de HB foi também realizada dividindo-se a população em quintis de peso (Tabela 3). Foi observada superestimação em todos os quintis, sendo que para o primeiro quintil a média de superestimação foi de $8 \%$, para o segundo quintil, de $7 \%$, para o terceiro quintil, de $7 \%$, para o quarto quintil, de $7,6 \%$ e, para o quinto quintil, foi de $4,5 \%$.

Foram elaboradas duas equações para cálculo do GMR: novo modelo e fator de correção para fórmula de HB.

O novo modelo obtido para os 180 casos com IMC acima de $35 \mathrm{~kg} / \mathrm{m}^{2}$ (Gl) foi:

GMR estimado $=172,19+10,93($ peso em $\mathrm{kg})+$ 3,10 (altura em cm) - 2,55 (idade em anos)

Para validação do modelo e comparação com a fórmula de $\mathrm{HB}$, efetuou-se a estimativa dos erros médios quadráticos sob as duas abordagens consideradas. Os resultados obtidos para a fórmula de $\mathrm{HB}$ e para a realidade brasileira foram iguais a 205,32 e 207,07 , respectivamente.

O novo modelo obtido para os 580 casos com IMC abaixo de $35 \mathrm{~kg} / \mathrm{m}^{2}$ (G2) foi: ATUAL

GMR estimado $=407,57+9,58($ peso $\mathrm{em} \mathrm{kg})+$ 2,05 (altura em cm) - 1,74 (idade em anos)

O mesmo cálculo de validação aplicado ao Gl foi aqui novamente aplicado e os resultados obtidos para a fórmula de $\mathrm{HB}$ e para a realidade brasileira foram iguais a 172,99 e 152,24 , respectivamente.

Ainda em busca de um melhor resultado para calcular o GMR, foi formulado um fator de correção para melhor ajuste da fórmula de $\mathrm{HB}$ à nossa população, por meio de um modelo de regressão, considerando-se o resultado da fórmula de $\mathrm{HB}$ como variável explicativa $\mathrm{e}$ o valor de GMR obtido pela CI como variável resposta.

Para o Gl, o fator de correção para fórmula de $\mathrm{HB}$ foi:

GMR estimado $=-268,80+1,1203(\mathrm{HB})$

Para o G2, o fator de correção para fórmula de $\mathrm{HB}$ foi:

GMR estimado $=87,05+0,8783(\mathrm{HB})$

Para o G1 e o G2, foi também calculado o erro médio quadrático para validação do fator de correção, sendo que, para o Gl, os resultados obtidos para a fórmula de HB e para a fórmula de HB com fator de correção foram iguais a 205,32 e 197,98, respectivamente. Já para o G2 os resultados obtidos para a fórmula de $\mathrm{HB}$ e para a fórmula de HB com fator de correção foram iguais a 172,99 e 154,86 , respectivamente.

Calculou-se também o coeficiente de correlação observado entre o GMR e o valor calculado pela fórmula de HB corrigida para G1 e para G2, respectivamente, de 0,737 e de 0,589 , indicando boa correlação entre os resultados (Figuras 2 e 3 ). 
Tabela 2. Caracterização do GMR de acordo com intervalos de peso (quintis)

\begin{tabular}{lccccccc}
\hline Quintis de peso $\mathbf{( k g})$ & $\mathbf{n}$ & Média (cal) & IC de 95\% para a média (cal) & Mediana (cal) & Mínimo (cal) & Máximo (cal) & Desvio-padrão \\
\hline Até 66 & 158 & $1.225,9$ & $(1.200,2-1.251,6)$ & 1.210 & 910 & 1.850 & 163,6 \\
66,1 a 73 & 149 & $1.334,5$ & $(1.311,7-1.357,3)$ & 1.330 & 1.070 & 1.740 & 141,0 \\
73,1 a 82 & 157 & $1.409,4$ & $(1.382,8-1.435,9)$ & 1.420 & 920 & 1.880 & 168,6 \\
82,1 a 93 & 146 & $1.512,7$ & $(1.478,1-1.547,3)$ & 1.500 & 1.040 & 2.420 & 211,6 \\
Mais de 93 & 150 & $1.775,6$ & $(1.726,1-1.825,1)$ & 1.725 & 1.190 & 3.400 & 306,9 \\
Geral & 760 & $1.448,7$ & $(1.428,9-1.468,5)$ & 1.420 & 910 & 3.400 & 278,2 \\
\hline
\end{tabular}

GMR: gasto metabólico de repouso; peso em quilos (kg); cal: calorias.

Tabela 3. Variação percentual entre o valor de GMR e 0 valor da fórmula de Harris Benedict (HB) de acordo com intervalos de peso (quintis)

\begin{tabular}{|c|c|c|c|c|c|c|c|}
\hline \multirow{2}{*}{$\begin{array}{l}\text { Classificação } \\
\text { quintis de peso (kg) }\end{array}$} & \multicolumn{7}{|c|}{ Variação \% (HB em relação ao GMR) } \\
\hline & $\mathbf{n}$ & Média (\%) & $\begin{array}{c}\text { IC de } 95 \% \\
\text { para a média }\end{array}$ & Mediana & Mínimo & Máximo & Desvio-padrão \\
\hline Até 66 & 158 & 8,0 & $(6-10,1)$ & 9,1 & $-36,7$ & 39,5 & 13,2 \\
\hline 66,1 a 73 & 149 & 7,0 & $(5,2-8,9)$ & 6,4 & $-23,4$ & 38,5 & 11,5 \\
\hline 73,1 a 82 & 157 & 7,0 & $(4,9-9,1)$ & 5,1 & $-21,5$ & 52,6 & 13,3 \\
\hline 82,1 a 93 & 146 & 7,6 & $(5,4-9,8)$ & 6,0 & $-33,3$ & 46,7 & 13,7 \\
\hline Mais de 93 & 150 & 4,5 & $(2,4-6,7)$ & 3,3 & $-22,0$ & 93,4 & 13,4 \\
\hline
\end{tabular}

GMR: gasto metabólico de repouso; peso em quilos (kg).

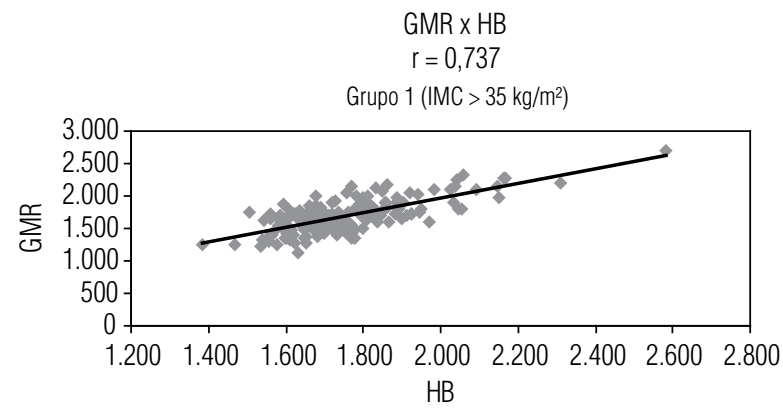

Figura 2. Correlação entre o gasto metabólico de repouso (GMR) e a fórmula de Harris e Benedict (HB) no grupo 1 (IMC $\geq 35 \mathrm{~kg} / \mathrm{m}^{2}$ ).

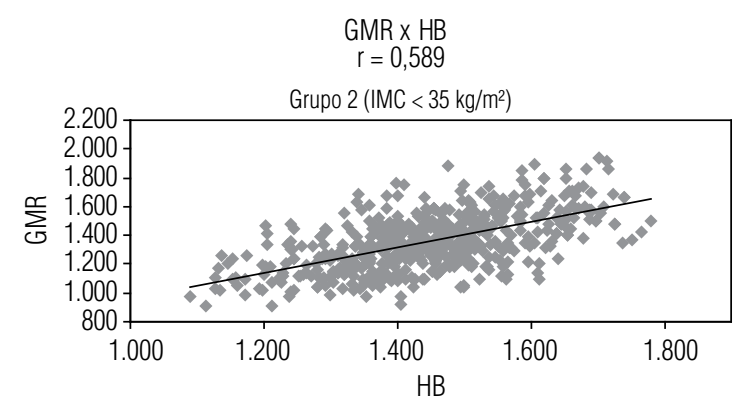

Figura 3. Correlação entre o gasto metabólico de repouso (GMR) e a fórmula de Harris e Benedict (HB) no grupo 2 (IMC < 35 kg/m²).

\section{DISCUSSÃO}

É consenso que uma ingestão calórica (IC) elevada e estilo de vida sedentário podem ocasionar um desequilíbrio no $\mathrm{BE}$, o que a longo prazo pode levar a aumento de peso importante (1). Quando a IC é maior que o GET, ocorre um BE positivo, o que favorece o aumento do estoque energético. A situação oposta leva a um BE negativo e consequente depleção do estoque energético (18). Considerando que o GMR é o principal componente do GET e que é a partir deste que o valor calórico final a ser indicado ao paciente é calculado a fim de proporcionar um BE negativo, essa superestimação, mesmo sendo pequena, pode ser prejudicial para o tratamento da obesidade $(3,6,7,19)$.

Mostramos, neste estudo, que a fórmula de HB tende a superestimar o GMR na população feminina brasileira, em torno de 7\%. Apesar de parecer pequena, essa superestimação pode levar a uma perda de peso menor em $20 \%$ ao mês, o que é bem significativo.

Nosso valor de superestimação encontra-se de acordo com o observado em outros estudos, em que foi observada uma superestimação de aproximadamente $4 \%$ a $5 \%(20,21)$.

As equações hoje utilizadas na prática clínica brasileira foram elaboradas, em sua maioria, baseadas em 
populações europeias e norte-americanas, que possuem realidade completamente diferente da nossa. As equações parecem ser populações específicas, sofrendo influência da etnia e do estado nutricional $(22,23)$.

Mulheres afro-americanas têm GMR menor que mulheres americanas de origem europeia, evidenciando, portanto, a influência da etnia no GMR (21).

O grau de sobrepeso é um fator de grande influência nas equações preditivas e vale lembrar que a maior parte das equações hoje utilizadas foi feita baseada em indivíduos sem sobrepeso ou obesidade (22).

Existe atualmente uma necessidade evidente de que as fórmulas propostas sejam elaboradas de acordo com o grau de obesidade. Entretanto, o uso da composição corporal parece não ter grande influência, apesar de a massa livre de gordura ser metabolicamente mais ativa. Logo, as novas equações propostas têm sido elaboradas de acordo com a classificação de IMC. Vale ressaltar que essa variável, além de ser uma medida simples que avalia o nível de adiposidade, tem grande correlação com a prevalência de componentes da síndrome metabólica, em especial de risco cardiovascular $(17,19,22,24,25)$.

Nossa avaliação da influência de cada variável mostrou que a idade possui uma correlação inversa com o GMR, ou seja, quanto maior a idade menor o GMR (Figura 4). Essa situação já era esperada, pois, como demonstrado anteriormente, essa é uma tendência, uma vez que o organismo passa a gastar menor quantidade de energia com a idade. Entre as justificativas para esse declínio, estão: alterações na composição corporal que influenciam diretamente o GMR como redução da massa magra, redução da atividade física, alteração na função tireoidiana e menor atividade do sistema nervoso simpático (26).

O QR é obtido a partir da relação entre volume de gás carbônico expirado e o de oxigênio inspirado, variáveis essas envolvidas nas reações realizadas pelo organis-

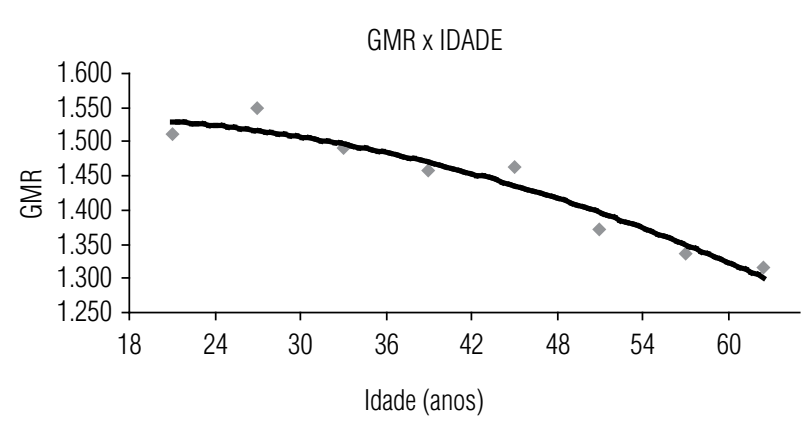

Figura 4. Correlação entre o gasto metabólico de repouso (GMR) e idade mo para produção de energia $(10,27)$. $\mathrm{O}$ valor do $\mathrm{QR}$ pode variar de 0,67 a 1,3. É possível, a partir do valor do $\mathrm{QR}$, avaliar qual tipo de substrato é prioritariamente utilizado pelo organismo para produção de energia, sendo que, quanto mais próximo de 0,7 for o resultado, maior é a oxidação de lipídios e, quanto mais perto de 1,0 , maior é a oxidação de carboidratos. Logo, quanto maior o $\mathrm{QR}$, maior é a chance de o paciente ganhar peso, pois nessa situação a oxidação de carboidratos é privilegiada em detrimento da oxidação de gorduras (28). Assim como evidenciado em nosso estudo, outros estudos mostram que o $\mathrm{QR}$ pode ser utilizado como um parâmetro preditor de obesidade, uma vez que indivíduos com um QR alto têm maior chance de ganhar peso ao longo dos anos quando comparados a indivíduos com QR menor, pois este apresenta uma correlação inversa com o GMR. Estudos mostram que mudanças no QR e GMR a longo prazo estão envolvidas em alterações do peso $(29,30)$.

Como explicado acima, um QR alto está relacionado com uma menor oxidação lipídica, o que implica risco para ganho de peso. Buscando estabelecer uma justificativa para as diferenças individuais na utilização de substratos energéticos, um estudo foi conduzido para avaliar a relação entre QR e a atividade do sistema nervoso simpático, uma vez que este tem um efeito estimulatório na oxidação lipídica, entretanto essa hipótese não foi comprovada (31).

A padronização do GMR mostrou que quanto maior o peso, maior é o GMR, sendo o peso a variável de maior influência no GMR, o que justifica a divisão da população de acordo com quintis de peso. Assim como nosso estudo, outros estudos mostraram que o peso é definitivamente a variável de maior influência, seguida pelo IMC, medida essa que tem relação direta com a prevalência de componentes da síndrome metabólica, em especial de risco cardiovascular $(17,21,23,24)$.

O uso do GMR como preditor de ganho de peso já é bem estabelecido (32).

A padronização do GMR apresentada neste estudo pode ser utilizada para determinar, de acordo com as faixas de peso, se o GMR obtido pela calorimetria encontra-se dentro do padrão de nossa população. Essa padronização pode ainda ser utilizada na prática clínica como referência para GMR quando este não puder ser aferido por CI.

Considerando que a fórmula de $\mathrm{HB}$ não é adequada para nossa população, como demonstrado em nossos resultados e por outros estudos, sugerimos duas novas 
opções de equações para estimação do GMR, dividindo a população de acordo com as faixas de IMC, pois, como explicado anteriormente, a necessidade dessa divisão ocorre porque a população com IMC acima de $35 \mathrm{~kg} / \mathrm{m}^{2}$ possui resultados discrepantes do resto da população, o que não permitiu a elaboração de um único modelo e tal divisão foi também adotada em outros estudos $(2,4,20,21,23,24)$.

Entre as duas opções propostas, o novo modelo de equação se mostrou superior à fórmula de $\mathrm{HB}$ com fator de correção e em relação à fórmula $\mathrm{HB}$ para população com IMC abaixo de $35 \mathrm{~kg} / \mathrm{m}^{2}$, uma vez que o erro médio quadrático foi menor para o novo modelo $(152,24,154,86$ e 172,99 , respectivamente). Já para a população com IMC acima de $35 \mathrm{~kg} / \mathrm{m}^{2}$, a melhor opção foi a fórmula de $\mathrm{HB}$ com fator de correção, uma vez que essa proposta foi a que apresentou o menor valor de erro médio quadrático quando comparado ao novo modelo e fórmula de HB $(197,98,205,32$ e 207,07, respectivamente).

Em populações extremamente obesas, é realmente mais difícil a adequação de fórmulas, pois existe uma dificuldade na escolha do peso a ser aplicado na fórmula, o que pode influenciar demasiadamente seus resultados. O uso do peso atual leva a uma superestimação dos resultados independentemente da fórmula a ser aplicada, e o uso do peso ideal ou ajustado pode levar a uma subestimação das necessidades (22). Outro ponto não favorável é o fato de que a maior parte das equações hoje utilizadas foi feita baseada em indivíduos sem sobrepeso ou obesidade e mesmo as fórmulas feitas com base em populações obesas parecem não se adequar e trazem resultados de GMR superestimados (33).

Além disso, o número de exames avaliados no Gl foi bem inferior que o do G2 (180 e 580, respectivamente), o que pode ter influenciado de forma negativa os resultados para nossa população de obesos.

A partir deste estudo foi possível padronizar e normatizar os valores de GMR para diferentes faixas de peso, para que estes possam ser usados como referência em nossa população.

Concluímos, a partir da correlação entre as variáveis, que quanto maior o peso, maior o GMR, sendo esta a variável de maior influência e, quanto maior a idade, menor o GMR, podendo o GMR assim como o QR serem utilizados como preditores de obesidade, tendo nossos valores de GMR padronizados como referência.

Além disso, mostramos que a fórmula de $\mathrm{HB}$ tende a superestimar os valores de GMR em nossa população e apresentamos duas novas opções de equações para estimativa do GMR de maneira mais precisa, sendo que, para população com IMC $\leq 35 \mathrm{~kg} / \mathrm{m}^{2}$, o novo modelo se aplicou melhor e, para população com IMC > 35 $\mathrm{kg} / \mathrm{m}^{2}$, o fator de correção foi mais preciso.

Declaração: os autores declaram não haver conflitos de interesse científico neste estudo.

\section{REFERÊNCIAS}

1. Winsier RL, Bracco D, Schuts Y. Predicit effects of small decreases in energy expenditure on weight gain in adult women. Int $\mathrm{J}$ Obes. 1993;693-700.

2. Warlich V, Anjos A. Aspectos históricos e metodológicos da medição e estimativa da taxa metabólica basal: uma revisão da literatura. Cad Saude Publica. 2001;17(4):801-7.

3. Suman OE, Mlcak RP, Chinkes DL, Herndon DN. Resting energy expenditure in severely burned children: analysis of agreement between indirect calorimetry and prediction equations using the Bland-Altman method. Burns. 2006;32(3):335-42.

4. Frankenfield D, Roth-Yousey L, Compher C. Camparison of predictive equations for resting metabolic rate in healthy nonobese and obese adults: systematic review. J Am Diet Assoc. 2005;105(5):775-89.

5. Ravussin E, Lilioja S, Knowler WC, Christin L, Freymond D, Abbot $\mathrm{W}$, et al. Reduced rate of energy expenditure as a risk factor for body-weight gain. N Eng J Med. 1988;318:467-72.

6. Warlich V, Anjos A. Aspectos históricos e metodológicos da medição e estimativa da taxa metabólica basal: uma revisão da literatura. Cad Saude Publica. 2001;17(4):801-7.

7. Frankenfield D, Roth-Yousey L, Compher C. Camparison of predictive equations for resting metabolic rate in healthy nonobese and obese adults: systematic review. J Am Diet Assoc. 2005;105(5):775-89.

8. Miller MD, Daniels LA, Bannerman E, Crotty M. Resting energy expenditure measured longitudinally following hip fracture compared to predictive equation: is an injury adjustment required? $\mathrm{Br}$ J Nutr. 2005;94(6):976-82.

9. Campbell CG. Predict vs. measured energy expenditure in critically ill, underweight patients. Nutr Clin Pract. 2005;20(2):276-80.

10. Compher C, Cato R, Bader J, Kinosian B. Harris-Benedict equations do not adequately predict energy requirements in elderly hospitalized African Americans. J Med Assoc. 2004;96(2):209-14.

11. De Lorenzo A, Tagliabue A, Andreoli A, Testolin G, Comelli M, Deurenberg $P$. Mesured and predict resting metabolic rate in Italian males and females, aged 18-59y. Eur J Clin Nut. 2001;55(3):208-14.

12. Luhrmann PM, Neuhaeuser BM. Are equations published in literature for predicting resting metabolic rate accurate for use in the elderly? J Nutr Health Aging. 2004;8(3):144-9.

13. Vasconcellos M. Fontes de inadequação das recomendações internacionais sobre requerimentos humanos de energia para a população brasileira. Rev Bras Epidemiol. 2002;5(1):59-72.

14. Warlich V, Anjos LA. Validação de equações de predição da taxa metabólica basal em mulheres residentes em Porto Alegre, RS, Brasil. Rev Saude Publica. 2001;35(1):39-45.

15. Suman OE, Mlcak RP, Chinkes DL, Herndon DN. Resting energy expenditure in severely burned children: analysis of agreement between indirect calorimetry and prediction equations using the Bland-Altman method. Burns. 2006;32(3):335-42.

16. Kien $C L$, Ugrasbul F. Prediction of daily energy expenditure during a feeding trial using measurements of resting energy ex- 
penditure, fat free mass, or Harris Benedict equations. Am J Clin Nutr. 2004;80(4):876-80.

17. Cercato C, Silva S, Sato A, Mancini M, Halpern A. Risco cardiovascular em uma população de obesos. Arq Bras Endocrinol Metabol. 2000;44(1):45-8.

18. Suplicy HL. Etiopatogenia da obesidade. Rev Bras Nutr Clin. 2000;15:290-3.

19. Rodrigues AE, Marostegan PF, Mancini MC, Dalcanale L, Melo ME, Cercato $\mathrm{C}$, et al. Análise da taxa metabólica de repouso avaliada por calorimetria indireta em mulheres obesas com baixa e alta ingestão calórica. Arq Bras Endocrinol Metab. 2008;52(1):76-84.

20. Frankenfield DC, Muth ER, Rowe WA. The Harris Benedict studies of human basal metabolism: history an limitation. J Am Diet Assoc. 1998;98:439-45.

21. Feet CA, Fett CRW, Marchinini JS. Gasto energético de repouso vs. estimado e relação com a composição corporal de mulheres. Arq Bras Endocrinol Metabol. 2006;50(6):1050-8.

22. Weg MWV, Watson JM, Klesges RC, Clemens LHE, Slawson DL, MCClanahan BS. Development and cross-validation of a prediction equation for estimating resting energy expenditure in healthy African-American and European-American women. Eur J Clin Nutr. 2004;58:474-80.

23. Weijs JMP. Validity of predictive equations for resting energy expenditure in US and Dutch overweight and obese class I and II adults aged 18-65y. Am J Clin Nutr. 2008;88(4):959-70.

24. Muller JM, Bosy-Westphal A, Klaus S, Kreymann G, Luhrmann PM, Neuhauser-Berthold $M$, et al. World Health Organization equations have shortcomings for predicting resting energy expenditure in persons from a modern, affluent population: generation of a new reference standard from a retrospective analysis of a German database of resting energy-expenditure. Am J Clin Nutr. 2004;80:1379-90.

25. Feitosa ACR, Mancini M, Cercato C, Villares S, Halpern A. Relação entre o perfil metabólico e níveis de leptina em indivíduos obesos. Arq Bras Endocrinol Metabol. 2007;51(1):59-64.

26. Frisard M I, Broussard A, Davies SS, Roberts II J, Rood J, Jonge $L$, et al. Aging, resting metabolic rate, and oxidative damage: results from the Louisiana Healthy Aging Study. J Gerontol A Biol Sci Med Sci. 2007;62(7):752-9.

27. Rocha E, Alves VGF, Fonseca RB. Indirecty calorimetry: methodology, instruments and clinical application. Curr Opin Clin Nutr Metab Care. 2006;9:247-56.

28. Stephen A, Mc Clave MD, Lowen CL, Kleber M, Mc Connell J, Junj L, et al. Clinical use of the respiratory quotient obtained from indirect Calorimetry. J Parenter Enteral Nutr. 2003;27(1):21-6.

29. Ravussin E. Metabolic differences and the development of obesity. Metabolism. 1995;44(Suppl 9):12-4.

30. Weyer C, Pratley RE, Salbe AD, Bogardus C, Ravussin E, Tataranni AP. Energy expenditure, fat oxidation and body weight regulation: a study of metabolic adaptation to long term weight change. J Clin Endocrinol Metab. 2000;85(3):1087-94.

31. Snitker S, Tataranni A, Ravussin E. Respiratory quotient is inversely associated with muscle sympathetic nerve activity. J Clin Endocrinol Metab. 1998;83(11):3977-9.

32. Tataranni PA, Harper IT, Parigi AD, Vozarova B, Bunt J, Bogardus C, et al. Body weight gain in free-living Pima Indians: effect of energy intake vs expenditure. Int J Obes Relat Metab Disord. 2003;27:1578-83.

33. Luis DA, Aller R, Izaola O, Romero E. Prediction equation of resting energy expenditure in a adult Spanish population of obese adult population. Ann Nutr Metab. 2006;50:193-6. 